\title{
Risk analysis and acceptability criteria
}

\author{
M. Guarascio, M. Lombardi, G. Rossi \& G. Sciarra \\ Safety Engineering "Sapienza", Rome University, Italy
}

\begin{abstract}
The basic concepts and tools for the Quantitative Probabilistic Risk Analysis are presented.

The rationale of the risk acceptability criteria is discussed in the case of rail tunnel safety in Italy.

Keywords: Quantitative Probabilistic Risk Analysis, risk acceptability criteria.
\end{abstract}

\section{Introduction}

In general, risk analysis is a risk assessment tool which initially has been developed to investigate the safety of potentially dangerous industrial processes (e.g. in the chemical industry) or potentially dangerous industrial plants (such as nuclear power plants). Nowadays Quantitative Probabilistic Risk Analysis is a powerful common tool in many sectors of engineering design and operating practice both industrial and civil. The application of risk analysis should help to establish a proactive safety strategy by investigating potential risks. This safety strategy was to replace merely experience-based concepts learned from incidents or accidents, that had already happened.

Generally speaking, risk analysis deals with potential negative consequences of events that could occur when considering a technical system in a social environment. The only option in such a situation is to develop a representative model of the risks associated to the system in question. As there exists an unlimited number of possibilities of how dangerous effects may develop it is impossible to take all possible situations into account; therefore the only investigation is restricted to a limited number of selected representative scenarios. For that reason it should always be kept in mind that risk analysis is a model relying on preconditions and assumptions and is not a copy of reality. Nevertheless risk analysis provides a much better understanding of risk-related processes than merely experience-based concepts may ever achieve. 
The characteristics of Quantitative Probabilistic Risk Analysis and its applications can be summarized as follows:

- Quantitative Probabilistic Risk Analysis is a systemic approach to analyse sequences and interrelations in potential incidents or accidents, considering the logic chain events, critical dangerous events and undesired consequences.

- Quantitative Probabilistic Risk Analysis makes the quantification of risks establishing the basis of a performance-based approach for the assessment of safety standards

Quantitative Probabilistic Risk analysis can be used:

- to check general consistency of safety planning;

- to choose between alternative design solutions;

- to demonstrate that safety standards are fulfilled, e.g. in the case of deviations from prescriptions;

- to optimize safety planning in terms of cost-effectiveness.

To guarantee safety for human beings is nowadays done in many countries on the basis of prescriptive regulations: A prescriptive requirement specifies particular safety features, actions, or programmatic elements to be included in the design of buildings as well as tunnels or industrial processes, as the means for achieving a desired objective. The implementation of these requirements has more or less to be done without considering the individual characteristics of a building or industrial process or a tunnel.

By contrast the process of a risk-based approach allows a structured, harmonised and transparent assessment of risks for an individual specific hazard situation including the consideration of the local risk conditions in terms of relevant influence factors, their interrelations and possible consequences of incidents for a specific case. Moreover, it allows one to come up with the best additional safety measures in terms of risk mitigation and enables a comparison of different alternatives. Although risk can be reduced to very low levels, this may be considerably expensive and inappropriate to the assets to be protected. A risk based cost/effectiveness analysis ensures that the money spent to reduce risk is allocated in such a way that an optimum level of safety is obtained. Thus a risk based cost/effectiveness analysis can be the basis to assure that for every reduction of a unit of damage exactly the same unit is invested in protection.

Hence, the risk-based approach in the context of safety management offers the opportunity to get a transparent and consistent approach to decision-making about risks and can be an appropriate supplement to the implementation of measures to respect the requirements of standards and guidelines. 
The implementation of Risk Analysis and Risk Assessment in the Risk Management process can be based on the following three elements:

Risk Analysis: Quantitative Probabilistic Risk analysis is concerned with the fundamental question: "What might happen and what are the consequences?" In the case of quantitative analysis probabilities of accidents and their consequences for different damage indicators (e.g. in terms of fatalities, injuries, property damage, interruption of services) and the resulting risk are estimated.

Risk Evaluation: Quantitative Probabilistic Risk evaluation is directed towards the question of acceptability and the explicit discussion of safety criteria. For a systemic and operable risk evaluation one has to define safety criteria and to determine whether a given risk level is acceptable or not. In other words risk evaluation has to give an answer to the question "Is the estimated risk acceptable?"

Risk and Safety Management: If the estimated risk is considered as not acceptable, additional safety measures have to be proposed. Therefore the effectiveness and also cost-effectiveness of different safety measures can be determined by using the initial frequency and consequence analysis of the scenarios which will be positively or negatively affected under the assumption that the investigated safety measure has been implemented. Planning of safety measures has to answer the question "Which measures are necessary to get a safe (and cost-efficient) system?"

\section{Basic of risk analysis methodology}

Hazardous conditions can develop from root events which evolve and generate an initiating critical event.

From this critical event several scenarios can develop according to the specific final hazard potential.

To each one hazard scenario can correspond several final consequence events according to the mode of occurrence and number of people involved.

The mentioned logical and sequential structure of events is graphically presented considering the "initiating critical event" as a "nodal point" separating the root events ("causes") and the consequence events ("effects").

The above described graph, usually named the Bow-Tie Model, is presented in fig. 1

The left hand side gives an account of the prevention activities aimed at the reduction of the probability of the initiating critical event.

The right hand side shows the mitigation of the severity of the consequences due to the effectiveness of the safety systems and rescue services (the so called safety requirements).

The probabilistic operating tools used are the statistical inference techniques and Fault Tree Analysis (FTA) for the estimation of the probability of the initiating events. 


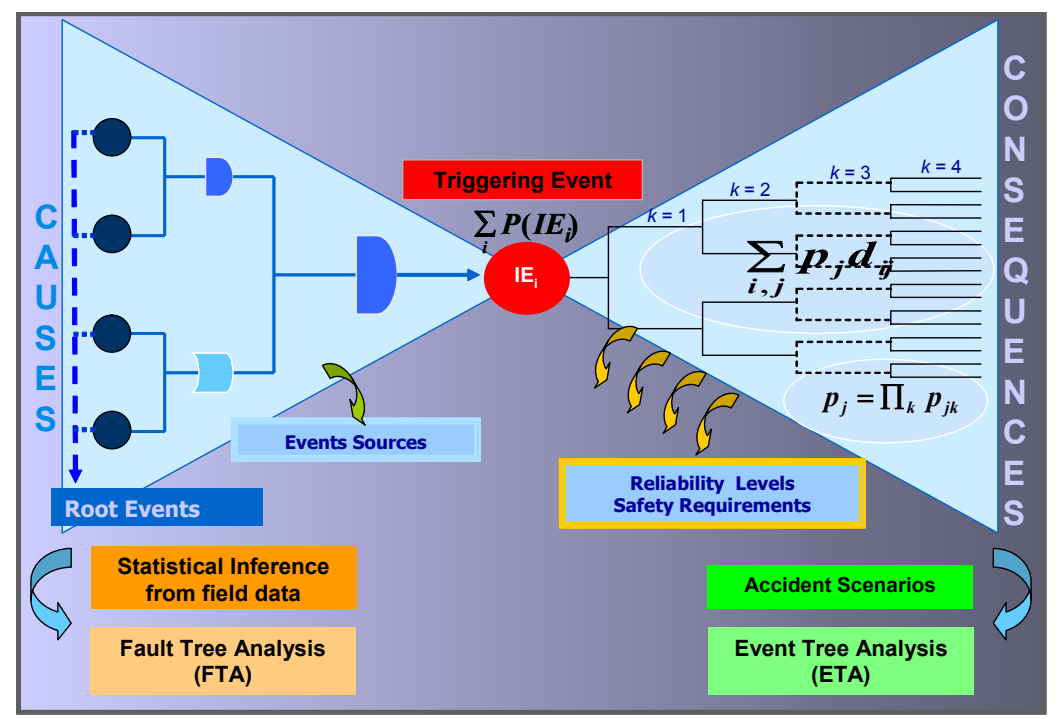

Figure 1: $\quad$ Bow-Tie Model: $P\left(\mathrm{IE}_{\mathrm{i}}\right)=$ Probability of the $i^{\text {th }} \mathrm{IE}_{\mathrm{i}}, \mathrm{p}_{\mathrm{j}}=$ Probability of the $\mathrm{j}^{\text {th }}$ hazard scenario, $\mathrm{p}_{\mathrm{jk}}=$ Reliability of the $\mathrm{k}^{\text {th }}$ safety requirement.

The Event Tree Analysis (ETA) for the estimation of the probabilities of all the final consequence events, where the severity is evaluated with the help of specific simulators reproducing the hazard scenario (computational fluid dynamics codes in case of fire hazard) as well as exodus and rescue scenarios.

The numerical results $\left(\boldsymbol{p}_{\boldsymbol{j} h}, \boldsymbol{d}_{\boldsymbol{j} \boldsymbol{h}}\right)$ obtained by the implementation of the methodology illustrated in figs. 1 and 2, namely the probabilities of the final consequences events together with the corresponding severity estimates (i.e. in terms of Number of Fatalities), are used to build up the various Risk Indicators according to given criteria [1].

Here below are defined the Risk Indicators to be used in Italy, according to the government act (2005) on "Safety in rail tunnels".

Individual Risk Indicators

- $\quad I R=$ Individual Risk (Probability to be "victim" in a "fatal" accident occurred in a specific tunnel in a year)

- $I R(x)=$ Point Individual Risk (Probability to be "victim" in a "fatal" accident occurred in a specific point $x$ of a tunnel in a year).

Societal Risk (Mode 1)

- $\quad R=$ Expected Risk Value (Fatalities/Year-Tunnel) 


\section{Societal Risk (Mode 2)}

- $B C R=$ Back Cumulated Risk Distribution so called FN curve (Probability $\mathrm{P}\left(\mathrm{N} \geq N_{t}\right) /$ Year-Tunnel)

where:

- $\mathrm{F}=$ Back Cumulated Probability

- $\mathrm{N}=$ Number of victims in a consequences event (Fatalities/Year-Tunnel)

- $N_{t}=$ Threshold value

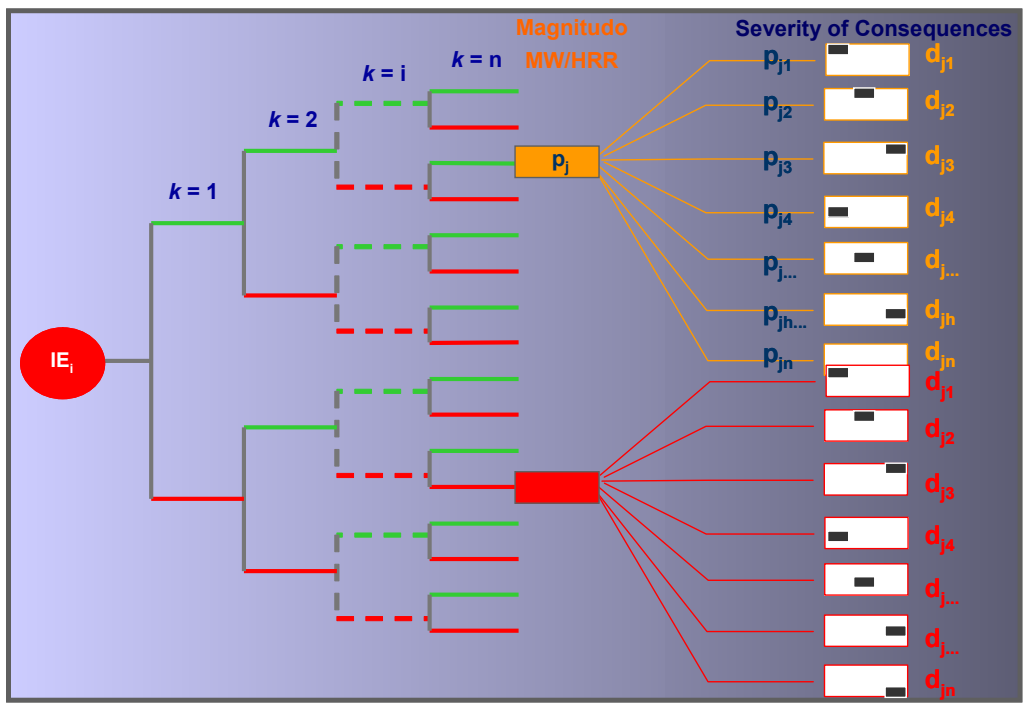

Figure 2: $\quad$ Event tree analysis: $p_{j h}=$ Probability of the $h^{\text {th }}$ consequences event given the $\mathrm{j}^{\text {th }}$ hazard scenario, $\mathrm{d}_{\mathrm{jh}}=$ Severity of the $\mathrm{h}^{\text {th }}$ consequences event given the $\mathrm{j}^{\text {th }}$ hazard scenario.

\section{Acceptability criteria}

The decision making on the level of safety to achieve in a given sector of activity (e.g. transport, industry) and consequently the level of risk to be accepted is undoubtedly a responsibility of the governmental authorities.

The hazard potential on one side and the technical as well as operational capability to face and manage the hazard scenarios on the other should back up the decision on what level of risk is to be considered acceptable.

The historical safety performance in similar environments could also be considered in order to identify an appropriate safety target.

In quantitative and numerical terms the acceptability criteria are defined by fixing a specific threshold when considering the risk indicators like $\boldsymbol{I R}, \boldsymbol{I R}(\boldsymbol{x})$ or $\boldsymbol{R}$. 
In the case of the $\boldsymbol{B C R}$ distribution the bilog representation plane F-N is used and a straight line is located at an appropriate position and slope with the role of a separation line between acceptability and unacceptability zones.

Here below are given examples of acceptability criteria included in the above mentioned governmental act in Italy regarding the "Safety in Rail Tunnels".

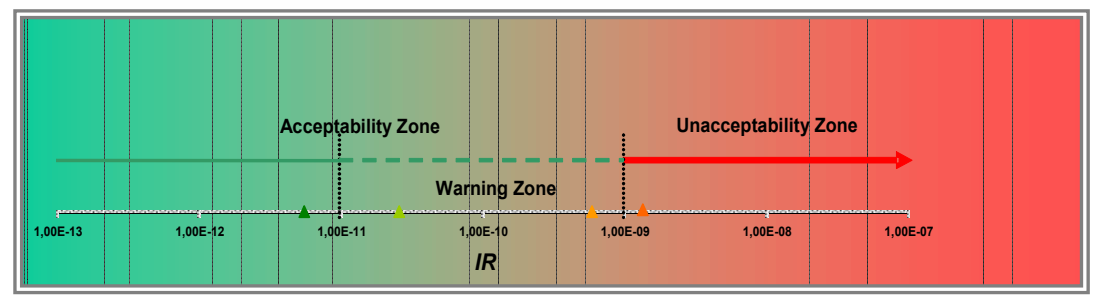

Figure 3: Individual risk acceptability criterion (Italian rail tunnels).

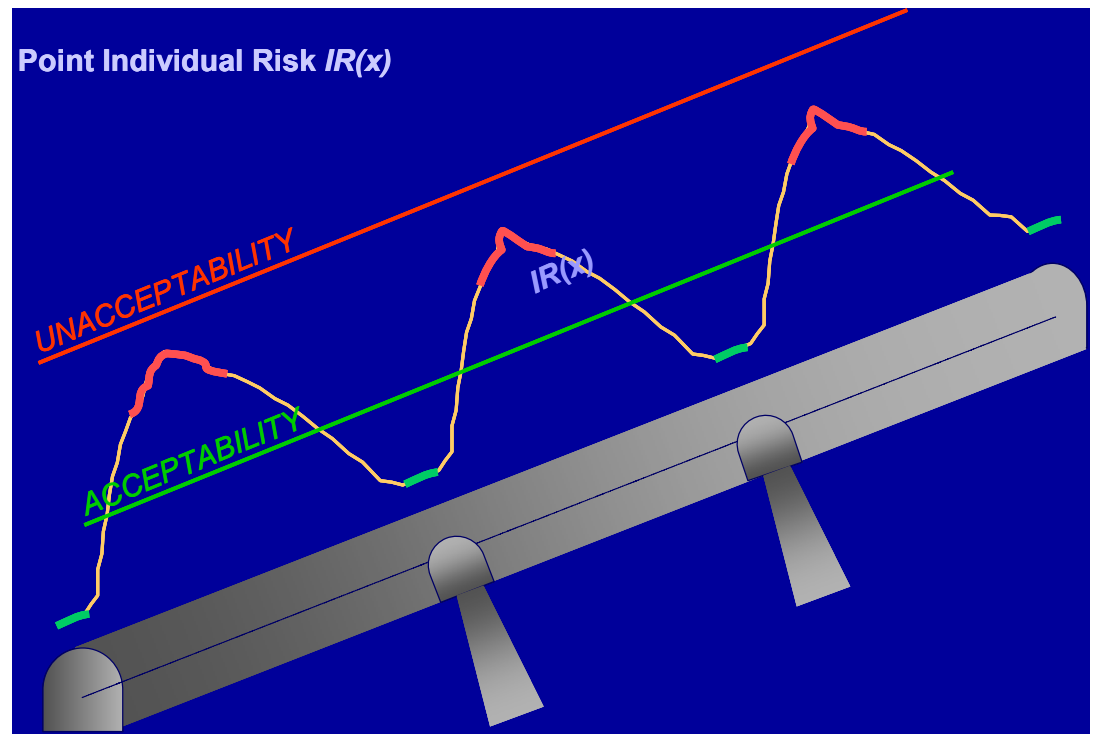

Figure 4: $\quad$ Point individual risk criterion (Italian rail tunnels).

\section{Conclusive remarks}

The Quantitative Probabilistic Risk Analysis methodology allows one to measure the level of risk using specific numerical indicators and then to compare then with threshold values according to appropriate acceptability criteria.

The aim and meaning of the various indicators and criteria reflect the relationships among government, authorities, owners or operators, users and people. 


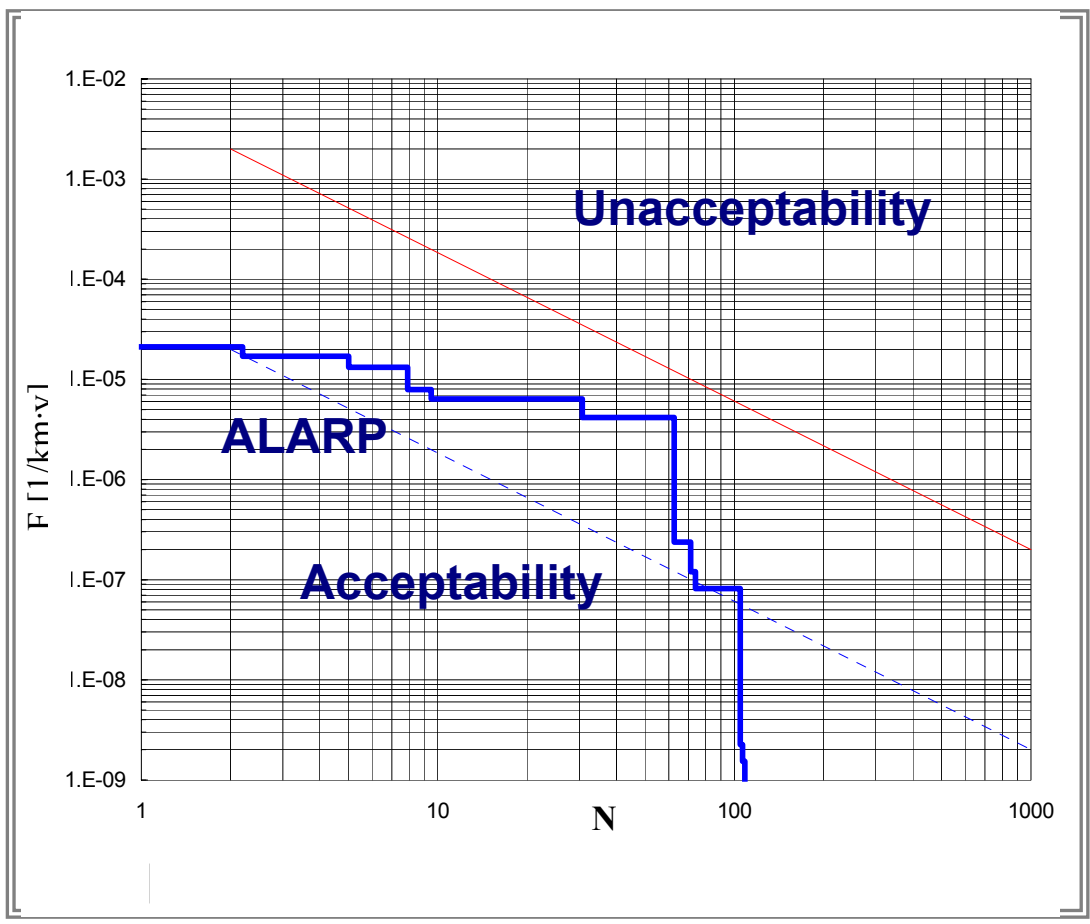

Figure 5: Societal Risk (Mode 2) acceptability criterion (Italian rail tunnels) an example of back cumulated risk distribution.

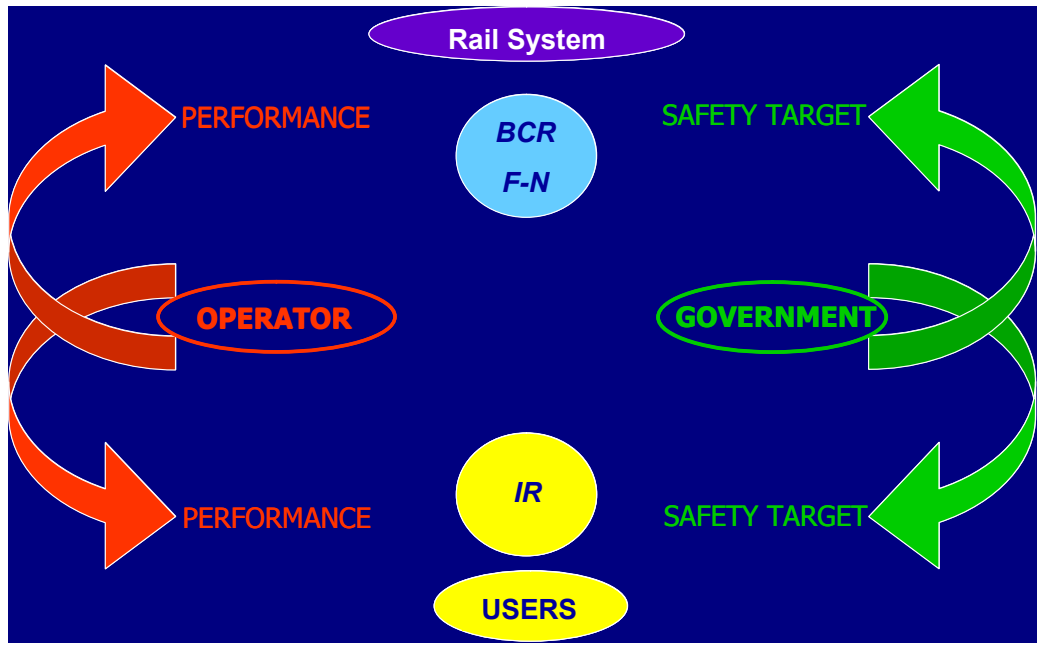

Figure 6: Rail safety tunnel targets and risk indicators. 
138 Safety and Security Engineering II

Fig. 6 schematically illustrates the specific use of $\boldsymbol{I R}$ and $\boldsymbol{B C R}$ in the framework of Rail Safety Tunnels where " $\boldsymbol{I R}$ " gives the measure of the safety performance that the operator is able to offer to the single user whereas " $\boldsymbol{B C} \boldsymbol{R}$ " defines the general level of the safety with respect to the target fixed by the government authorities.

\section{References}

[1] Guarascio, M. et al., Road tunnels safety rules in Italy: the tunnel country, Malta, Safe, 2007

[2] EU Directive 54/2004/CE, On minimum safety requirements for tunnels in the trans-European road network

[3] D. M. 28.10.2005, Rail Tunnel Safety 